ledge, as well as words, to teach what he professes to understand.

little further on in this treatise, he says :-

"All the valuable instruction I ever received was from a woman in the town where I lived, who had practised as a midwife for twenty years; she gave me more useful instruction in an interview of about twenty minutes, than all I ever gained from any other source."

Now, sir, I ask, what are we to think of a system founded alone on rash and reckless experiment, by $"$ man who acknowledges himself indebted to such a source for all the valuable information he possesses on a subject in which is involved human life and happiness.

Now, Mr. Speaker, in conclusion, I ask the members of this house, this grave and intelligent assembly, whether they are prepared by any act of legislative sanction, to recommend to their fellow citizens and the world, this pretended system of medicine, with all its boasting pretensions, its inperfections and preposterous absurdities, and all its abuse and vile slander, intended to prejudice the mind of the people against the regularly educated physicians, and which I do contend is as much a part of this system, as steaming and red pepper.

Sir, are we prepared to pronounce the experience of all those who have devoted their lives and talents to the study and practice of medicine, for the last five thousand years, under a deep sense of the responsibility under which they acted, entirely worthless? Are we prepared to usher a host of iguorant, boasting pretenders upon community, without the guarantee that they know even a part of that imperfect system which they pretend to practise, utterly ignorant of the human system and the diseases which affect it, and destitute of every qualification but the proof of having paid twenty dollar's for a Thomsonian book ?

Sir, let us pass this bill or any similar one, and we do all this. I am now willing to submit this subject to the intelligence, justice and bumanity of this grave assembly, and take my seat.

\title{
a CASE OF CROUP.
}

BY F. A. WILLARD, M. D.-

[Coinmunicnted for the Boston Medical and Surgical Journal.]

In conmunicating to my professional brethren, through the medium of your excellent Journal, the following case of croup, my only object is, to endeavor to draw their attention to a disease, which, after having advanced to a certain stage, is perhaps as little under the control of medicine as tubercular phthisis, and seems to be nearly as great a scourge to children in northern climates as the other is to adolescents.

I was called, March $15 \mathrm{th}$, to visit $\mathbf{T}-\mathbf{C}-$, a boy three years old, who had been remarkably healthy up to the time of his present indisposition. I found him laboring under the following symptons;pulse one hundred and thirty, hard, full, quick, and rebounding; breathing laborious, suffocative, and performed with a kind of bissing unise; voice shrill, as if the sound passed through a brazen tube ; cough short, dry, and hard ; tongue red, swollen and indented; skin hot and dry, except 
the head and face, which were covered with perspiration from the violence of the struggle; lips and cheeks alternately pale and red; laying upon his back; neck considerably engorged; head thrown back so as to keep the trachea upon the stretch ; eyes protuberant ; countenance exhibiting great distress ; at times delirious. The above symptoms would seem to announce to the most superficial observer an aggravated case of the croup.

Having only a week previously, lost a patient laboring under similar symptoms and a similar disease, and having gone through the routine of remedies usually applied on such occasions, without any other result than that of relieving for the moment the most urgent symptoms, and perhaps delaying for a short time the fatal termination, I resolved, on being called to this patient, to deviate somewhat from the beaten track, and to follow up a mure energetic and persevering course of treatment ; and although the little sufferer finally sunk under the disease, yet it will appear that this result was delayed nine days; and may we not hope, that, by careful and reiterated observation, we may be able to discover some remedy for a disease which has been, and still is, as much an opprobrium medicina as any other to which human nature is heir to ?

Sunday, March 15th, at five o'clock, P. M. I was called to visit the patient, and found him laboring under the symptoms as stated above. I directed six leeches to be applied to the lower part of the trachea; five grains of submuriate of mercury to be given once in three hours; two drops of Scheel's prussic, or hydro-cyanic acid, to be taken once in four hours ; mild mercurial ointment to be rubbed over the groins; a warm bath once in six hours; to be kept constantly nauseated, and occasionally vomited by a solution of tartarized antimony.

March 16 h. - Passed an exceedingly restless night, but somewhat relieved this morning ; cough humid ; expectorates a small quantity of coagulable lymph, combined with fibrin or mucus; countenance exhibits great distress; pulse continues hard and quick; four more leeches to be applied to the trachea; hydro-cyanic acid to be continued; skin hot and dry ; bath as yesterday.

17 th. - The antimony and mercury have operated powerfully upon the bowels, the dejections being of a dark green color; the inflammatory action much diminished; expectorates more freely; a blister to be applied over the upper part of the thorax and the lower part of the trachea.

18th.-Passed a more comfortable night; expectoration increased, and small films, resembling portions of the membrane, coughed up; cough less, and more full; the following draught to be given every five hours :

R. Potassæe Nitratis grana quinque.

Aquæ Menthe pulegii drachmas quatior.

Vini Antimonii Turtariz. guttas quinque.

Tincturso Digitalis guttas quinque.

Mucilaginis Âcaciæ.

Syripi Sing. drachmam.

Solution of tartarized antimony to be discontinued ; sinapisms to the feet. 19th.- Much improved; mercurial fetor observable ; submuriate of mercury and mercurial ointment to be discontinued; expectoration more 
mucous, or rather purulent, combined with scarcely any fibrin or coagulable lymph; pulse ninety-eight, full and soft ; desires food.

20 th. - Sitting (1) in bed; slept during balf of the night ; expectoration copious; skin moist; the engorgement and fulness of the neck loave entirely disappeared; respiration free from that peculiar hissing which I believe is always observable in the acute inflammatory stage of this disease ; mouth sore from the influence of the mercury; desires food, is allowed thin arrow root, and gruel; much debilitated.

21 st.-Slept well; coughs occasionally, and expectorates freely large quantities of thick mucus; is clamorous for more food; warm bath discontinued ; skin moist and cool ; pulse eiglıty-nine ; the draught discontinued, as was also the hydro-cyanic acid yesterday; much annoyed by the blister. The little patient now appears to be convalescent, and there seems to be nothing remaining of the disease but debility.

22d.-The patient much emaciated, considering the time he has been ill ; appetite large; tongue slightly conted in the centre; pulse eighty, soft, and small; a small quantity of æhther to be given once in four hours, and the arrow root continued.

23d.-This forenoon, at eleven o'clock, I was sent for in haste, to see the patient, and found him in articulo mortis; upon his back; face and lips pale and livid; insensible to surrounding objects; cough sliort, quick, and suffocative, and these symptoms continued to increase in severity until one o'clock, when he died.

On interrogating the mother, I learned that the patient slept well during the nighi; that at six o'clock she arose and washed the child's neck and face with cold water ; that at about seven, the child was seized with horripilations, soon became "stuffed up," skin became hot, the respiration impeded, and all these symptoms augmented in severity until his death.

I have thus, Mr. Editor, stated, in as condersed a form as I was able, the symptoms and treatment of the disease, and which were noted down in my case book at the bedside of the patient.

Alihough $I$ an very well aware that it is not customary to record in our journals unfavorable cases, yet it appears to me that where there is in them anything that is novel, they should be unhesitatingly and ingenuously made known.

Charlestown, April 27, 1835.

\section{CIRONIC APHTHA.}

\section{To the Editor of the Boston Medical and Surgical Journal.}

Sik,-On page 420, Vol. XI. of your practical Journal, is a notice of the effects of Secale Cornutum, by Dr. Jonathan Sivett. He ascribes a disease called Chronic Aphthæ to its administration. I have seen a similar affection to the one described, in cases where no ergot bad ever been exhibited. I would therefore respectfully inquire of $\mathrm{Dr}$. S. if he can throw more light on so important a subject. I should be pleased to see a full account from him of the disease in question, and also the proper treatment. I should be pleased, also, to see from any of your corres- 